\title{
Construction of Hybrid Deep Learning Model for Predicting Children Behavior based on their Emotional Reaction
}

\author{
Dr. T. Senthil Kumar, \\ Associate Professor, \\ Computer Science and Engineering Department, \\ Amrita Vishwa Vidyapeetham, \\ Coimbatore, India. \\ t_senthilkumar@cb.amrita.edu
}

\begin{abstract}
Emotion prediction, the sub-domain of sentiment analysis helps to analyze the emotion. Recently, the prediction of children's behavior based on their present emotional activities is remaining as a challenging task. Henceforth, the deep learning algorithms are used to support and assist in the process of children's behavior prediction by considering the emotional features with a good accuracy rate. Besides, this article presents the prediction of children's behavior based on their emotion with the deep learning classifiers method. To analyze the performance, decision tree and naïve Bayes probability model are compared. Totally, 35 sample emotions are considered in the prediction process of deep learning classifier with a probability model. Furthermore, the hybrid emotions are incorporated in the proposed dataset. The comparison between both the decision tree and the Naïve Bayes method has been performed to predict the children's emotions after the classification. Based on the probability model of naïve Bayes method and decision tree, naïve Bayes method provides good results in terms of recognition rate and prediction accuracy when compared to the decision tree method. Therefore, a fusion of these two algorithms is proposed for predicting the emotions involved in children's behavior. This research article includes the combined algorithm mathematical proof of prediction based on the emotion samples. This article discusses about the future scope of the proposal and the obtained prediction results.
\end{abstract}

Keywords: Deep learning, human emotion recognition

Information Technology

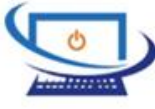


Journal of Information Technology and Digital World (2021)

Vol. 03/ No. 01

Pages: $29-43$

https://www.irojournals.com/itdw/

DOI: https://doi.org/10.36548/jitdw.2021.1.004

\section{INTRODUCTION}

In the artificial intelligence domain, human behavior prediction is one of the challenging problems. Many research works are assuming the participants rationally based on the cognitive process. Child development will be predicted by incorporating machine learning as a scientific study for observing the pattern of growth [1]. The change and stability of the children's growth are based on conception through adolescence. Figure 1 shows some sample images from children's unknown emotions activities.

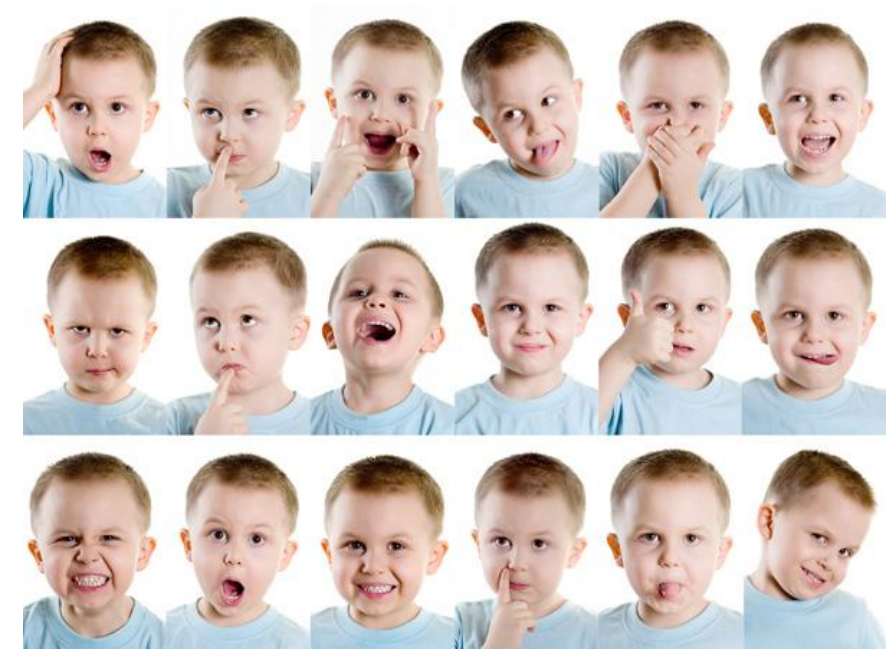

Figure 1 Sample unknown emotions from children

Generally, this growth will be based on biology, anthropology, sociology, education, and psychology behaviours of the participants [2]. Recently, this growth study has increased for any reason with the help of artificial intelligence methods. Because the human life success, health and emotional can be settled up with their roots in early childhood [3]. Figure 2 shows human emotion's valence band chart.

Information Technology

\&

Digital World 
Journal of Information Technology and Digital World (2021)

Vol. 03/ No. 01

Pages: $29-43$

https://www.irojournals.com/itdw/

DOI: https://doi.org/10.36548/jitdw.2021.1.004

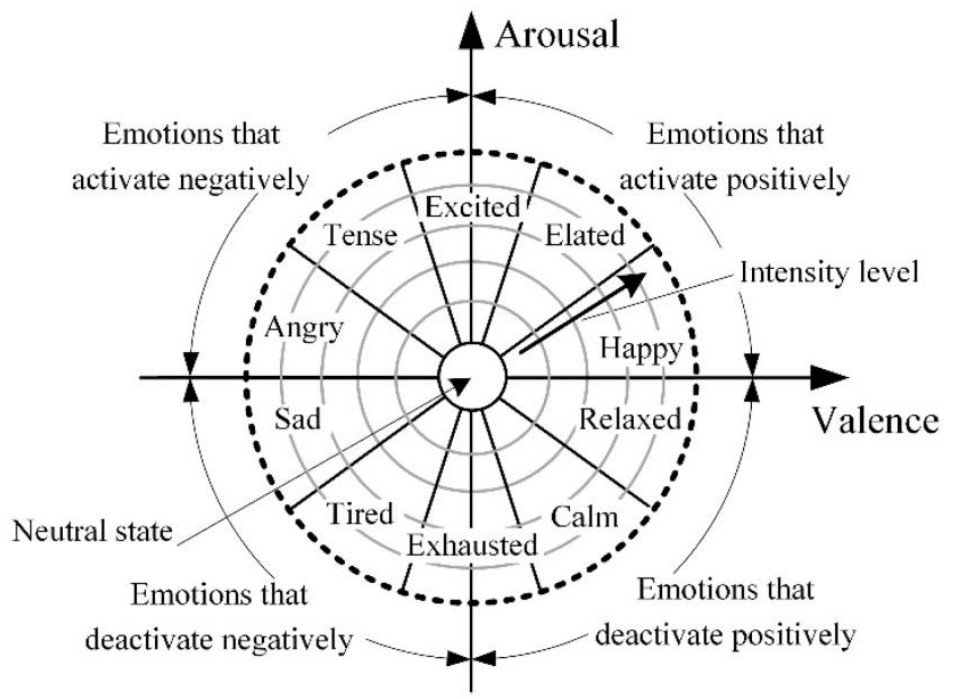

Figure 2 Human Emotion's Chart

The demonstration of the deep learning algorithm is enhanced for obtaining more predictive success with feature processing. This learning representation can be adopted for more flexible models [4]. The basic knowledge of the input in particular network construction is a cautious design choice to learn the feature processing. The feed-forward network is represented with the convolution function, which will provide more effective visualization testing [5]. The low-level feature derivation for the pixels can be done by using linear prediction with small input invariant function. Figure 3 shows some symbolic pictorial representation of human emotion [6].
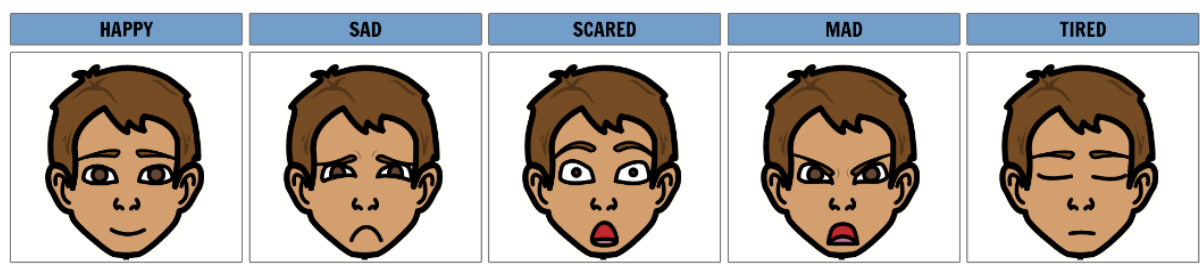

Figure 3 Samples of symbolic representation of human's emotions

The learning results should be improved with the estimation of longer observation of the participants in the evaluation process. Usually, this evaluation is achieved through some basic questionnaires [7]. The collection of a dataset is used for training and estimating activities with 
Journal of Information Technology and Digital World (2021)

Vol. 03/ No. 01

Pages: $29-43$

https://www.irojournals.com/itdw/

DOI: https://doi.org/10.36548/jitdw.2021.1.004

the participants present within the learning activities [8][9][10]. The participant's engagements are having a quantitative and qualitative measurement of emotion based on the behavioural and cognitive aspects. Their attention can be classified with many factors of emotional conditions [11][12][13][14].

\section{ORGANIZATION OF THE RESEARCH}

The proposed research article is constructed with several sections; section 3 discusses about the recent research works and section 4 contains the proposed prediction methodology on emotional activities by using the deep learning algorithm. Section 5 includes a discussion with obtained results. The conclusion and future scope of the proposed research are presened in section 6 .

\section{PRELIMINARIES}

Ross Quinlan a researcher proposes machine learning techniques for child behavioural prediction. The decision tree algorithm is used for the support system and it has been proved that, it is faster and easier than other existing classification problems [15]. The decision representation is very simple and the prediction rate is very high. The accuracy of the classifiers refers to the class label of the rule extraction dataset. The application-oriented tools are different and rough dataset-based methods are newer alternatives. The computational time of decision trees are simple and very short for the extended rules obtained from the algorithm is facilitated.

Clark et al discuss invariant rotation modeling for the general architecture of the solution space, which is included for obtaining the good solution. This work is similar to behavior theory setting and prediction-based prior assumptions can lead the deep learning identification will be encoded [16].

Masuyama et al proposed the cumulative contribution of 32 various emotions in a testing experiment involved with 19 participants with prediction rating. They achieved good results with the various fundamental emotion lists. Some of the multi-agent applications are solved by

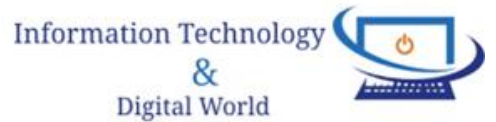


Journal of Information Technology and Digital World (2021)

Vol. 03/ No. 01

Pages: $29-43$

https://www.irojournals.com/itdw/

DOI: https://doi.org/10.36548/jitdw.2021.1.004

utilizing the deep learning model [17]. Storkey et al and silver et al work in identifying the action of any sportsman. They classify the emotions from the description of the dataset. This setting in a dataset is fixed and stable and finally it forms normal distribution [16] [18]. Based on the size of the network's input, the convolutional networks are getting some invariant property to the stationary function with similar layers with convolution. Lin et al construct the network model for game-theoretic invariance property. The detection of the emotion from their speech is a challenging task that contains many features to use. Some common issues in the detection of emotions are discussed in this research article [10].

1. Use of features based on what are emotions.

2. Use of signals for segments in the networks.

3. Type of learning methods should be noted to detect the emotions.

Chuang 2004 et al prove that, the emotions predicted from the speech are based on pitch and formants. The difference between happiness and anger is indicated through the scale. The energy in the speech can be considered for the classification in order to provide improved results at the final stage. The segregation of the energies for different emotions is tabulated and used for measurement. The fixing of the scale depends on various human emotions. Usually, the happiness energy will be higher than sadness. And anger or surprise energy will be greater than the energy of fear [19]. The research paper discusses the spectral features with emotion detection. They used Mel frequency cepstral coefficient (MFCC) methods to detect emotions. The data analysis is based on the model scale of the speech processing involved in the deeper networks. The contrast of MFCC frequency bands is mapped on the scale. This can be analyzed for identifying the speech information. This co-efficient is analyzed through cosine Fourier transform formulae of the speech signal [20]. Also some other research article proposes emotion detection based on the length of the speech segments. The various segments are extracting the information from signals and it concludes the emotional parameters [21].

Abdullah et al identified some sentiments and emotions from Arabic tweets by using a convolutional neural network- long short-term memory (LSTM) learning algorithm. They proposed and succeeded after conducting two-phase test by involving the feed-forward phase model and CNN-LSTM phase model respectively [22]. Chatterjee et al conducted some

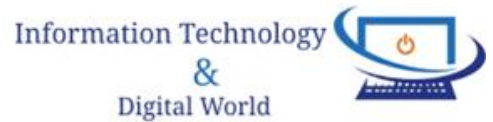


Journal of Information Technology and Digital World (2021)

Vol. 03/ No. 01

Pages: $29-43$

https://www.irojournals.com/itdw/

DOI: https://doi.org/10.36548/jitdw.2021.1.004

emotional contextual from their speech dialog delivery and classification has been performed. Their classes include various emotion classes like happy, sad, fear, angry and it is classified by LSTM. They are compared with many techniques with the help of measurement metrics [23].

\section{METHODOLOGIES}

Generally, the decision trees work with two data mining methods namely classification tree and regression tree [24]. The classification tree is used to predict the outcome of the data and the regression tree analysis method is used to predict the outcome based on the numerical data. Also this research work has conducted two exams, such as case 1 and case 2 . In case 1 , the proposed prediction classifier has been operated alone for performing classification and prediction. The performance of the proposed system has been tested and measured. But, in the case 2 ; the algorithm has been proposed for prediction along with the hybrid function in the fusion format of two classifiers named as decision tree and naïve Bayes algorithm. Figure 4 shows the workflow of proposed algorithm.

\section{Case 1:}

Emotions are predicted with separate classifiers

Let us consider this data,

$$
(x, Y)=\left(x_{1}, x_{2} \ldots . x_{k}, Y\right)
$$

The expected gain can be calculated and the entropy is defined as,

$$
H(T)=I_{E}\left(P_{1}, P_{2} \ldots P_{J}\right)=\sum_{i=1}^{J} P_{i} \log _{2} P_{i}
$$

Expected gain $=$ entropy - sum of entropy

Averaging over the possible with predicted model,

$$
=-\sum_{i=1}^{J} P_{i} \log _{2} P_{i} \sum_{a} p(a) \sum_{i=1}^{J}-P_{r}(i / a) \log _{2} P_{r}(i / a)
$$

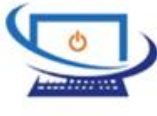


Journal of Information Technology and Digital World (2021)

Vol. 03/ No. 01

Pages: 29-43

https://www.irojournals.com/itdw/

DOI: https://doi.org/10.36548/jitdw.2021.1.004

The expected information gain is mentioned with an average and reduction of the entropy. Figure 4 shows the work flow of proposed framework.

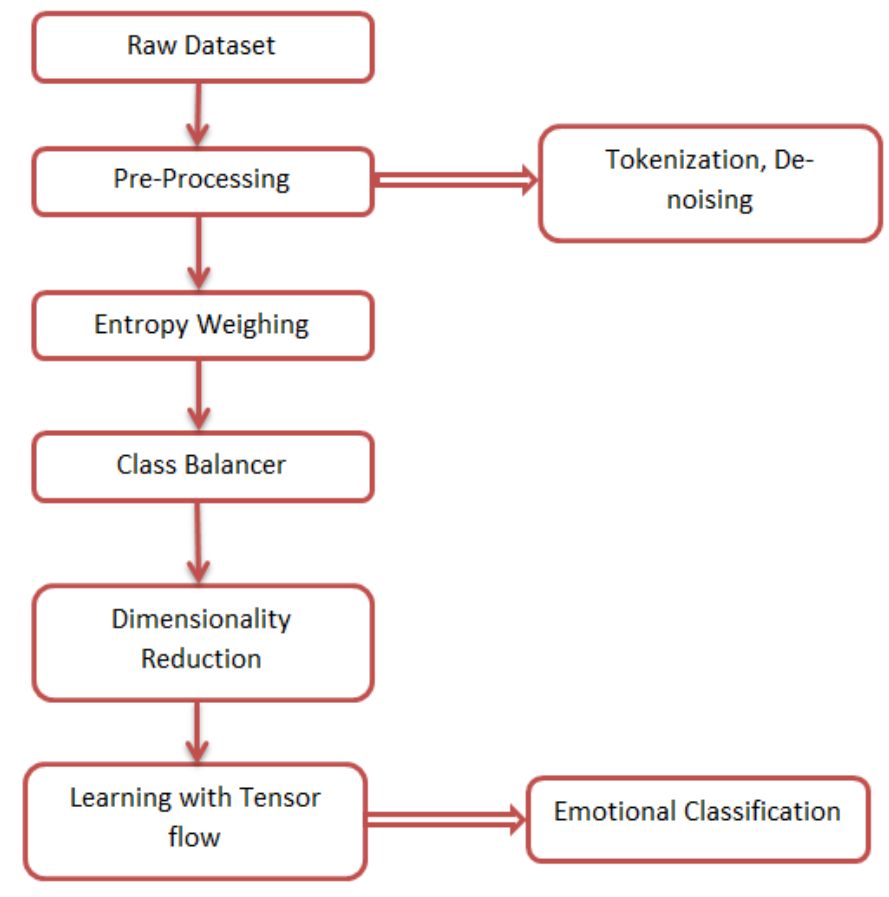

Figure 4 Proposed Framework

\section{Case 2:}

Emotion are predicting with fusion of two algorithm,

\section{Step 1:}

The estimation function,

$$
P\left({ }^{t_{j}} / y_{j}\right)=P\left(y_{j}\right) \prod P\left(\frac{x_{i j}}{y_{j}}\right)
$$

Step 2:

Expecting gain,

Expect $\left(\mathrm{x}_{\mathrm{j}}\right)=$ entropy - averaging over the possible values

Step 3:

Update the class value for each vector with prior probabilities, 
Journal of Information Technology and Digital World (2021)

Vol. 03/ No. 01

Pages: $29-43$

https://www.irojournals.com/itdw/

DOI: https://doi.org/10.36548/jitdw.2021.1.004

$$
P\left(y_{j} / t_{i}\right) ; y_{j}=y_{i} \rightarrow p\left(\frac{y_{j}}{t_{i}}\right)
$$

Step 4:

Again classifying with training samples with updated class values denoted as " $D$ ",

Step 5:

Split dataset, D into sub-data $\left\{D_{1}, D_{2} \ldots D_{N}\right\}$ depends on $x_{i}$

Step 6:

Calculate prior $P\left(y_{j}\right) \&$ Conditional $P\left(\frac{x_{i j}}{y_{j}}\right)$ probabilities of each sub-dataset $D_{i}$

Step 7:

Classify the prediction parameter of each dataset with their respective prior \& conditional probabilities.

Step 8:

For example, any sub data sets $\left(D_{i}\right)$ are misclassified; again re-calculation should be performed for the expected gain of attributes in that subset $D_{i}$

Step 9:

Continue this process, where examples are correctly classified.

All the prior probabilities for every dataset are classified with a minimum error rate and maximum accuracy, which is shown in the results and discussion section.

\section{RESULTS DISCUSSION}

The combined approach gives more interesting results and manages complexity in a good manner. The decision tree concepts are allowing the classification for an object by testing it with another classifier's properties [25]. The naïve Bayes method is a simple probabilities model, which is based on the Bayesian theorem. This can be suited for high dimension input data to process the classification. The proposed algorithm includes 1250 data, which are rich in emotion content for performing the extraction. These are labeled with 6 emotional categories. Figure 5 shows some sample images in dataset for the prediction of emotions.

Information Technology 
Journal of Information Technology and Digital World (2021)

Vol. 03/ No. 01

Pages: $29-43$

https://www.irojournals.com/itdw/

DOI: https://doi.org/10.36548/jitdw.2021.1.004

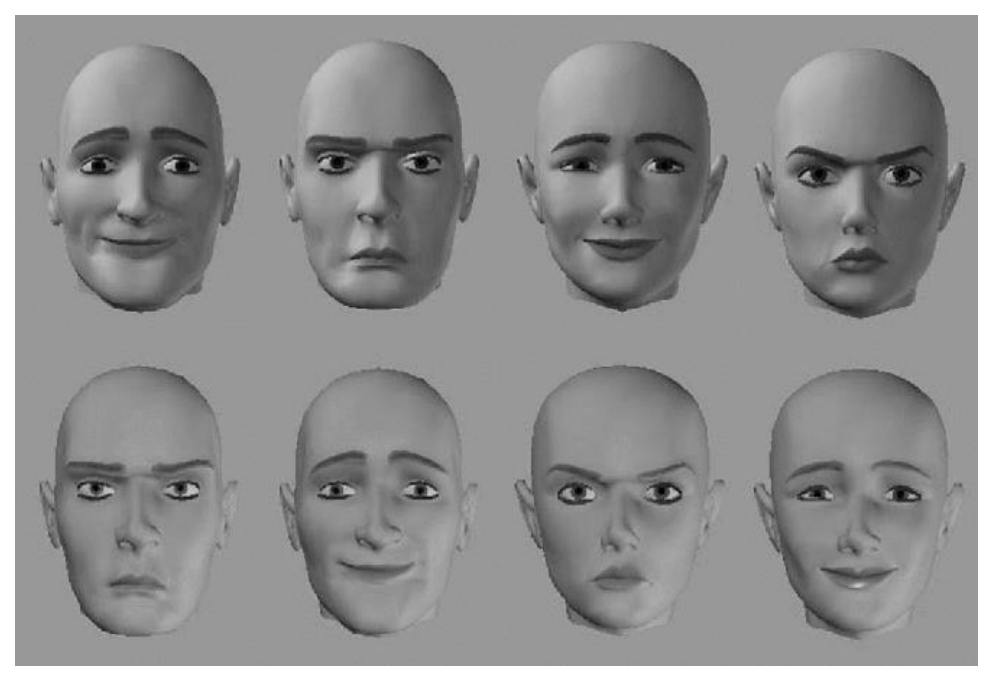

Figure 5 Sample Images for Prediction of Emotion

Figure 6 shows the normal distribution of single learning algorithm. The proposed algorithm is implemented after it gets tested and it is working well for predicting the emotions. This research work has taken only surprise, sorrow, fear, anger, and happy emotions.

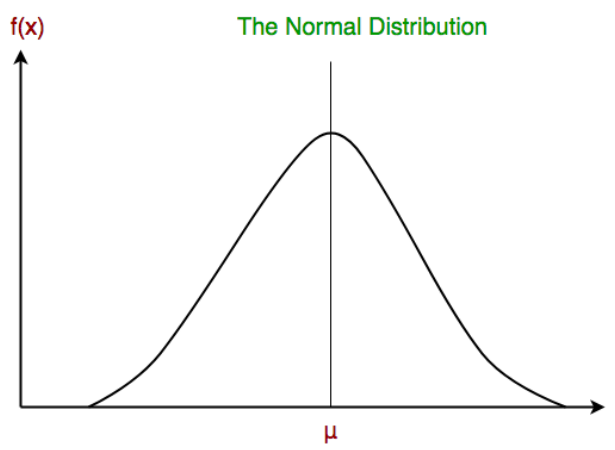

Figure 6 Normal Distribution of Single Learning Algorithm

The mean of the training images is obtained after comparing the distances between the mean images [26]. It is proved that, the proposed algorithm provides the best prediction results for all emotions compared to the individual algorithm used in prediction. 
Journal of Information Technology and Digital World (2021)

Vol. 03/ No. 01

Pages: $29-43$

https://www.irojournals.com/itdw/

DOI: https://doi.org/10.36548/jitdw.2021.1.004

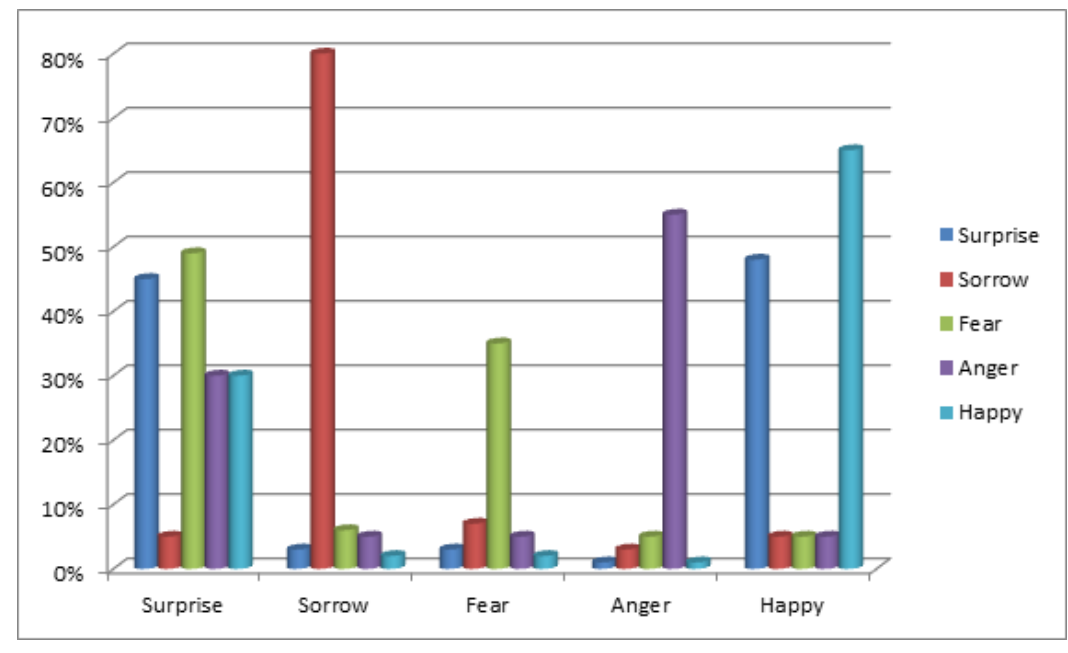

Figure 7 Recognition Rate of Single Type Classifier

Figure 7 shows the recognition rate of single type classifier. The individual algorithm approach based recognition rate is very low when compared to the fused algorithm approach [27]. When the hybrid algorithm has provided a $92 \%$ recognition rate for surprise emotion, another approach is providing less recognition rate and wrong prediction also.

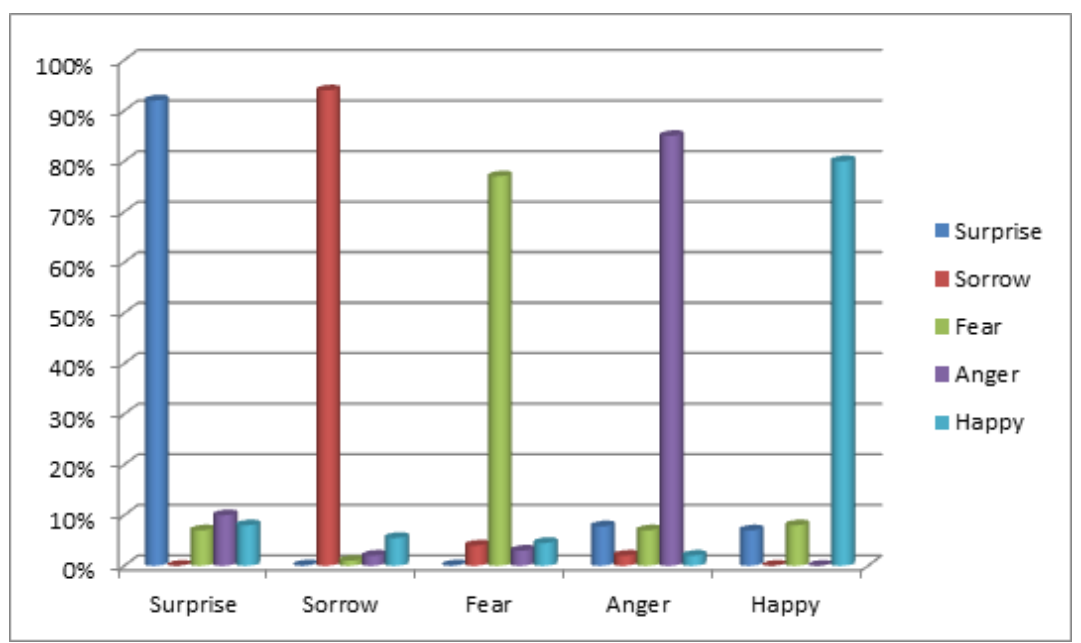

Figure 8 Recognition rate of proposed hybrid algorithm

Figure 8 shows that the recognition rate of proposed algorithm. It is proved that, the proposed algorithm is superior to the existing algorithms. Besides, a stronger training process has been 
Journal of Information Technology and Digital World (2021)

Vol. 03/ No. 01

Pages: $29-43$

https://www.irojournals.com/itdw/

DOI: https://doi.org/10.36548/jitdw.2021.1.004

taken for this improvised model. Table 1 shows the recognition rate values of the single learning approach. From these two graphs, we can conclude that our proposed hybrid techniques are providing good recognition rate for all emotions with high accuracy.

Table 1 Recognition rate of single learning approach

\begin{tabular}{|c|c|c|c|c|c|}
\hline & Surprise & Sorrow & Fear & Anger & Happy \\
\hline Surprise & $45 \%$ & $3.00 \%$ & $3.00 \%$ & $1.00 \%$ & $48 \%$ \\
\hline Sorrow & $5 \%$ & $8.3 \%$ & $7 \%$ & $3 \%$ & $5 \%$ \\
\hline Fear & $49 \%$ & $6 \%$ & $35 \%$ & $5 \%$ & $5 \%$ \\
\hline Anger & $30 \%$ & $5 \%$ & $5 \%$ & $55 \%$ & $5 \%$ \\
\hline Happy & $30 \%$ & $2.00 \%$ & $2.00 \%$ & $1 \%$ & $65 \%$ \\
\hline
\end{tabular}

Some measuring metrics are accuracy, sensitivity, recall have been used for measuring the performance of our proposed framework. Table 2 shows the performance of our proposed model. We can compare these two tables and can conclude that the hybrid learning method is holding a better recognition rate for emotion prediction.

Table 2 Recognition rate of hybrid learning approach

\begin{tabular}{|c|c|c|c|c|c|}
\hline & Surprise & Sorrow & Fear & Anger & Happy \\
\hline Surprise & $92 \%$ & $0.10 \%$ & $0.10 \%$ & $7.80 \%$ & $7 \%$ \\
\hline Sorrow & $0 \%$ & $94 \%$ & $4 \%$ & $2 \%$ & $0 \%$ \\
\hline Fear & $7 \%$ & $1 \%$ & $77 \%$ & $7 \%$ & $8 \%$ \\
\hline Anger & $10 \%$ & $2 \%$ & $3 \%$ & $85 \%$ & $0 \%$ \\
\hline Happy & $8 \%$ & $5.50 \%$ & $4.50 \%$ & $2 \%$ & $80 \%$ \\
\hline
\end{tabular}

\section{CONCLUSION}

Thus, the proposed research study conducts various case studies and testing for classification with hybrid type deep learning process. This hybrid prediction analysis is based on

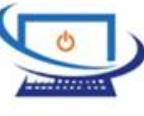


Journal of Information Technology and Digital World (2021)

Vol. 03/ No. 01

Pages: $29-43$

https://www.irojournals.com/itdw/

DOI: https://doi.org/10.36548/jitdw.2021.1.004

emotions that combination of naïve Bayes and the decision tree technique. This framework provides high accuracy and highly sensitive for the given prediction target. These techniques are providing good performance in terms of recognition rate, prediction accuracy, time consumption, and error rate. By a combination of these techniques, we can achieve more accurate prediction analysis. In our studies, the decision trees are having a big scale database and the naïve Bayes approach provides a prior probability prediction model for the proposed framework. Many attributes are the same for the process and it is the essential independent database. The proposed framework is categorizing the emotion-based human behavior across the application. The test can be conducted this both classification with step function with multiple classes. This emotion magnitude prediction is analyzed through a non-linear classification model with entropy assumption and it is proved best compared to a separate classification function. Our proposed framework can be very robust and small changes or updates of training data in the dataset acceptable for the final predictions. But our method will create complex over-fitting when big changes in training data which will be solved in future work [28]. In the future, our proposed model can be extended to classify the important and non-important emotions that should be considered from the real-time video stream.

\section{REFERENCES}

[1] Pandita, A.; Sharma, D.; Pandita, D.; Pawar, S.; Tariq, M.; Kaul, A. Childhood Obesity: Prevention is better than Cure. Available online: https://www.dovepress.com/childhood-obesityprevention-is-better-than-curepeer-reviewed-article-DMSO (June 2020).

[2] Fox, C.K.; Ryder, J.R.; Gross, A.C.; Kelly, A.S. Obesity in children and adolescents. In Obesity; Sbraccia, P., Finer, N., Eds.; Endocrinology; Springer International Publishing: Cham, Switzerland, 2019; pp. 295-322. [CrossRef]

[3] Anderson, Y.C.;Wynter, L.E.; Treves, K.F.; Grant, C.C.; Stewart, J.M.; Cave, T.L.;Wouldes, T.A.; Derraik, J.G.B.; Cutfield, W.S.; Hofman, P.L. Assessment of health-related quality of life and psychological well-being of children and adolescents with obesity enrolled in a New Zealand community-based intervention programme: An observational study. BMJ Open 2017, 7, e015776. [CrossRef] [PubMed]

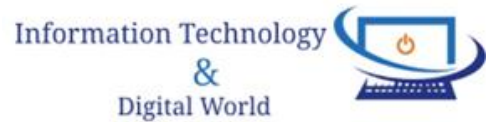


Journal of Information Technology and Digital World (2021)

Vol. 03/ No. 01

Pages: $29-43$

https://www.irojournals.com/itdw/

DOI: https://doi.org/10.36548/jitdw.2021.1.004

[4] S. A. Morsy and A. Rafea, "Improving document-level sentiment classification using contextual valence shifters", In Lecture Notes in Computer Science, No. 7337, 2012, pp.253258.

[5] R. A. Calvo and S. M. Kim, "Emotions in text: dimensional and categorical models", Computational Intelligence, vol. 29 no. 3, 2013, pp.527-543.

[6] K. Koroveshovski and S. Gievska, "Someone to talk to", In Proceedings of the Fifth Internation Conference on Applied Human Factors and Ergonomics AHFE 2014, vol. 19, 2014.

[7] D. Markovikj, S. Gievska, D.S. Stillwell, and M. Kosinski, "Mining facebook data for predictive personality modeling", In Proceedings of WCPR'13 (Shared Task), in conjunction with the Seventh international Conference on Weblogs and Social Media ICWSM'13, AAAI Press, 2013.

[8] S. Gievska and K. Koroveshovski, "The impact of affective verbal content on predicting personality impressions in YouTube videos", In Proceedings of the Workshop on Computational Personality Prediction 2014, Shared Task, in conjusction with ACM Multimedia 2014. (in press) [9] J-I. Biel and D. Gatica-Perez, "The YouTube lens: Crowdsourced personality impressions and audiovisual analysis of Vlogs. IEEE Transactions on Multimedia, vol.15, no. 1, 2013, pp. 41-55.

[10] M. Wöllmer, B. Schuller, F. Eyben and G. Rigoll, Combining Long Short-Term Memory and Dynamic Bayesian Networks for Incremental Emotion-Sensitive Artificial Listening, In: IEEE Journal of Selected Topics in Signal Processing, vol. 4, 2010, pp.867-881.

[11] Dada EG, Bassi JS, Chiroma H, Abdulhamid SM, Adetunmbi AO, Ajibuwa OE. Machine learning for email spam filtering: review, approaches and open research problems. Heliyon 2019;5(6):e01802. https://doi.org/10.1016/j.heliyon.2019. e01802.

[12] Xie M. Development of artificial intelligence and effects on financial system. J Phys Conf 2019;1187:032084. https://doi.org/10.1088/1742-6596/1187/3/032084.

[13] Hegazy O, Soliman OS, Salam MA. A machine learning model for stock market prediction. Int J Comput Sci Telecommun 2014;4(12):16-23.

[14] Beckmann JS, Lew D. Reconciling evidence-based medicine and precision medicine in the era of big data: challenges and opportunities. Genome Med 2016;8(1):134-9. 
Journal of Information Technology and Digital World (2021)

Vol. 03/ No. 01

Pages: $29-43$

https://www.irojournals.com/itdw/

DOI: https://doi.org/10.36548/jitdw.2021.1.004

[15] Quinlan J.R., 1986. Induction on decision trees, Machine learning, 1(1):81-106.

[16] C. Clark and A. J. Storkey. Training deep convolutional neural networks to play go. In Proceedings of the 32nd International Conference on Machine Learning, ICML 2015, 2015.

[17] Masuyama, Eitaro., "A number of fundamental emotions and their definitions", Robot and Human Communication, RO-MAN '94 Nagoya, Proceedings of the 3rd IEEE International Workshop, 156-161, (1994)

[18] D. Silver, A. Huang, C. J. Maddison, A. Guez, L. Sifre, G. van den Driessche, J. Schrittwieser, I. Antonoglou, V. Panneershelvam, M. Lanctot, S. Dieleman, Grewe D, J. Nham, N. Kalchbrenner, I. Sutskever, T. Lillicrap, M. Leach, K. Kavukcuoglu, T. Graepel, and D. Hassabis. Mastering the game of go with deep neural networks and tree search. Nature, 529, 2016.

[19] Chuang, Ze-Jing \& Wu, Chung-Hsien, "Multimodal Emotion Recognition from Speech and Text", Computational Linguistics and Chinese Language Processing, Vol. 9, No. 2, pp. 45-62, August, (2004).

[20] M. Gera and S. Goel, "A model for predicting the eligibility for placement of students using data mining technique," International Conference on Computing, Communication and Automation, vol. 4, pp. 18-23, January 2015.

[21] Rankin, J.; Matthews, L.; Cobley, S.; Han, A.; Sanders, R.; Wiltshire, H.D.; Baker, J.S. Psychological Consequences of Childhood Obesity: Psychiatric Comorbidity and Prevention. Available online: https://www.dovepress.com/psychological--consequences-of-childhoodobesity-psychiatric-comorbidipeer-reviewed-article-AHMT (June 2020).

[22] Abdullah M, Hadzikadicy M, Shaikhz S. SEDAT: sentiment and emotion detection in Arabic text using CNN-LSTM deep learning. Paper presented at: Proceedings of the 2018 17th IEEE International Conference on Machine Learning and Applications; 2018:835-840; IEEE.

[23] Chatterjee A, Gupta U, Chinnakotla MK, Srikanth R, Galley M, Agrawal P. Understanding emotions in text using deep learning and big data. Comput Hum Behav. 2019;93:309-317.

[24] Weber GM, Mandl KD, Kohane IS. Finding the missing link for big biomedical data. Jama 2014;311(24):2479-80.

Information Technology \& Digital World 
Journal of Information Technology and Digital World (2021)

Vol. 03/ No. 01

Pages: $29-43$

https://www.irojournals.com/itdw/

DOI: https://doi.org/10.36548/jitdw.2021.1.004

[25] Loconsole C, Chiaradia D, Bevilacqua V, Frisoli A. Real-time emotion recognition: an improved hybrid approach for classification performance. Intelligent Computing Theory 2014:320-31.

[26] Huang X, Kortelainen J, Zhao G, Li X, Moilanen A, Sepp€anen T, Pietik€ainen M. Multimodal emotion analysis from facial expressions and electroencephalogram. Compute Vis Image Understand 2016;147:114-24. https://doi.org/10.1016/j. cviu.2015.09.015.

[27] Bhattacharya J, Lindsen JP. Music for a brighter world: brightness judgment bias by musical emotion. PloS One 2016;11(2):e0148959. https://doi.org/10.1371/ journal.pone.0148959.

[28] Sangaiah AK, Arumugam M, Bian G-B. An intelligent learning approach for improving ECG signal classification and arrhythmia analysis. Artif Intell Med 2019: 101788. https://doi.org/10.1016/j.artmed.2019.101788. 